\title{
Application of wearable EEG sensors for indoor thermal comfort measurements
}

\author{
Silvia Angela Mansi ${ }^{1}$, llaria Pigliautile², Camillo Porcaro ${ }^{3,4}$, Anna Laura Pisello², Marco Arnesano ${ }^{1}$ \\ ${ }^{1}$ Università Telematica eCampus, 22060 Novedrate (CO), Italy \\ ${ }^{2}$ CIRIAF - Centro interuniversitario sull'inquinamento e I'ambiente Mauro Felli, Dipartimento di Ingegneria, Università di Perugia, \\ 06125 Perugia, Italy \\ ${ }^{3}$ Department of Neuroscience and Padova Neuroscience Center (PNC), University of Padova, Padova, Italy \\ ${ }^{4}$ Institute of Cognitive Sciences and Technologies (ISTC) - National Research Council (CNR), Rome, Italy
}

\begin{abstract}
Multidomain comfort theories have been demonstrated to interpret human thermal comfort in buildings by employing human-centered physiological measurements coupled with environmental sensing techniques. Thermal comfort has been correlated with brain activity through electroencephalographic (EEG) measurements. However, the application of low-cost wearable EEG sensors for measuring thermal comfort has not been thoroughly investigated. Wearable EEG devices provide several advantages in terms of reduced intrusiveness and application in real-life contexts. However, they are prone to measurement uncertainties. This study presents results from the application of an EEG wearable device to investigate changes in the EEG frequency domain at different indoor temperatures. Twenty-three participants were enrolled, and the EEG signals were recorded at three ambient temperatures: $\operatorname{cold}\left(16^{\circ} \mathrm{C}\right)$, neutral $\left(24^{\circ} \mathrm{C}\right)$, and warm $\left(31^{\circ} \mathrm{C}\right)$. Then, the analysis of brain Power Spectral Densities (PSDs) was performed, to investigate features correlated with thermal sensations. Statistically significant differences of several EEG features, measured on both frontal and temporal electrodes, were found between the three thermal conditions. Results bring to the conclusion that wearable sensors could be used for EEG acquisition applied to thermal comfort measurement, but only after a dedicated signal processing to remove the uncertainty due to artifacts.
\end{abstract}

Section: RESEARCH PAPER

Keywords: Thermal comfort measurement, electroencephalography (EEG); wearable sensors, signal processing

Citation: Silvia Angela Mansi, Ilaria Pigliautile, Camillo Porcaro, Anna Laura Pisello, Marco Arnesano, Application of wearable EEG sensors for indoor thermal comfort measurements, Acta IMEKO, vol. 10, no. 4, article 33, December 2021, identifier: IMEKO-ACTA-10 (2021)-04-33

Section Editors: Carlo Carobbi, University of Florence, Gian Marco Revel, Università Politecnica delle Marche and Nicola Giaquinto, Politecnico di Bari, Italy Received October 7, 2021; In final form December 6, 2021; Published December 2021

Copyright: This is an open-access article distributed under the terms of the Creative Commons Attribution 3.0 License, which permits unrestricted use, distribution, and reproduction in any medium, provided the original author and source are credited.

Funding: This work was supported by the Italian Ministry of Research through the NEXT:COM (Prot.20172FSCH4) "Towards the NEXT generation of Multiphysics and multidomain environmental COMfort models: theory elaboration and validation experiment" project, within the PRIN 2017 program.

Corresponding author: Marco Arnesano, e-mail: marco.arnesano@uniecampus.it

\section{INTRODUCTION}

Human-building interaction is an essential subject studied in the last decades, aimed at ensuring the occupants' wellbeing and the correlated influence on buildings' energy consumption. An important aspect of the built environment is indoor thermal comfort and its impact on occupants. From the 1970s, different thermal comfort models have been introduced. Firstly, the twonode model [1] was presented by J. B. Pierce, which represents the human body into two-layer: skin and core. Each layer is a heat transfer node with thermal physiological parameters controlled by energy and mass conservation low. Then, Fanger [2] proposed the Predicted Mean Vote (PMV) model to predict the thermal comfort of a large sample of individuals. In the last 3 decades, the adaptive model was widely used [3]. It is based on findings from the simultaneous collection of data on the thermal environment and thermal response of subjects, to determine the indoor thermal states, and the influencing parameters, that satisfy occupants' sensations. The resulting adaptive models provide experimental relationships between the thermal comfort temperature and the outdoor air temperature. However, neither of those models encompass the personal physiological and psychophysics influence on the individual thermal perception. The concept of the personal comfort model has been introduced as a novel approach to predict individual-specific thermal comfort based on the measurements of environmental quantities, occupants' behaviour, and physiological responses. 
The usage of different physiological signals has been discussed in the literature, in the field of thermal comfort measurements. Four of them have been identified as highly correlated with the perceived thermal comfort: electroencephalogram (EEG), electrocardiogram (ECG), skin temperature (ST), and galvanic skin response (GSR), being a part of different processes involved in the thermoregulatory system activities [4]. Several studies evaluated the thermal status of individuals acquiring different physiological signals simultaneously, but analysed them separately [5]. Only a few of those provide a regression equation that relates physiological parameters to each other [6].

In addition, wearable sensors, for measuring real-time physiological signals, have been assessed as a promising technology in the field of personal thermal comfort estimation [7]. Among physiological signals, measurable via wearable devices, the EEG has instigated interest, in the field of thermal comfort, for the possibility to monitor the human physiological responses changes in real-time [8].

Generally, the brain's electrical activity changes in response to the process of perception and cognition of environmental stimuli. EEG represents the measure of voltage fluctuation resulting from ionic current within the neurons of the brain [9]. Brainwaves are detected using sensors placed on the scalp according to the 10-20 system [10]. They are divided into bandwidths. Each of those corresponds to a particular state of mind. In general, delta $(0.1 \mathrm{~Hz}-4 \mathrm{~Hz})$ is associated with deep sleep, theta waves $(4 \mathrm{~Hz}-7.5 \mathrm{~Hz})$ are related to consciousness sleep towards drowsiness, alpha waves $(7.5 \mathrm{~Hz}-12 \mathrm{~Hz})$ are the prominent rhythm in relaxing and passive attention activities, beta $(12 \mathrm{~Hz}-30 \mathrm{~Hz})$ is associated with active thinking and gamma waves $(30 \mathrm{~Hz}-45 \mathrm{~Hz})$ are prominent during high mental activities.

Recent studies showed how EEG power spectral densities (PSDs) were influenced by changes in environmental temperature. Lv and colleagues [11] correlated the EEG frequency bands to the neutral and warm air temperatures. They showed higher delta-band activity in the warm condition. Yao and colleagues [10] measured EEG signals from 20 subjects exposed at low, neutral, and high temperatures, showing that the relative EEG power of the beta band was significantly higher in the cold and warm environment compared with the neutral condition. Lim et al. [12] found a connection between the EEG and thermal comfort, alpha/beta ratio (RAB) increased in a comfortable environment, the opposite trend was highlighted by the relative beta (RB). Other studies [12], [17] revealed how EEG frequencies change in conjunction with body temperature variations and how the ambient temperature influences EEG PSDs. Son et al. [13] investigated a correlation between psychological and physiological measures to evaluate thermal comfort. Their results showed an increase of theta band and a decrease of the beta band according to the thermal pleasure. Wu et al. [14] classified thermal comfort under different conditions, showing an increase of delta and a decrease of beta power in a warm environment. Zhu [15] examined changes in EEG responses during cognitive activity at different air temperatures. Findings indicated a high value of relative delta and a low value of relative theta, alpha, and beta at high temperatures. In the above-mentioned EEG studies, measurements were performed using a traditional cap or medical devices; these devices can provide good quality of data, but they require a long time of applications and often they are perceived as unpleasant by the users. Moreover, they are not applicable in a real-life context. Such issues could be solved by the advent of wearable sensors.
They offer several advantages: they are low cost, simple to be used, their comfortable design allows to reduce the time of application and, more importantly, they considerably attenuate the obstructiveness of measurements, making the experimental time not unpleasant for the participants. However, they are strongly prone to collect environmental noise and artifacts due to subject movements (e.g., eyeblink, muscular artifacts), which means that acquired data need to be processed before they became reliable for thermal comfort measurement [21]. Several studies performed a metrological characterization of wearable devices for testing their accuracy. Arpaia et al. [8] proposed a human stress detection method based on EEG signal acquired by a highly wearable single channel instrument. The results of their study demonstrated that the four standard machine learning (ML) classifiers used, reached more than $90 \%$ of accuracy in distinguishing stress conditions of participants. In another study, Arpaia and colleagues [16] present the calibration and the metrological characterization of a low-cost wearable device (Olimex EEG-SMT). Preliminary calibration results showed good linearity, however, a magnitude of error around $8 \%$ with a dependence on frequency was detected. In general, studies revealed how the commercial low-cost wearable devices used in conjunction with ML classifiers in an experimental context, can reach an accuracy between $83.3 \%$ and $99.1 \%$ [17]. Thus, findings revealed that EEG wearable sensors can provide the required accuracy for the classification of human mental states.

This paper presents the application of EEG wearable sensors for thermal comfort measurement. The experimental protocol, the signal processing procedure, and the statistical analysis are illustrated together with results from the measurement campaign performed in a controlled environment. Results demonstrate the feasibility of the proposed approach, that could be used to build personalized comfort models based on EEG measurement.

\section{MATERIALS AND METHODS}

\subsection{EEG data collection and processing}

\subsubsection{EEG measurement device}

In this study, the EEG signal acquisition was done using a commercial wearable device: the Interaxon MUSE headband [18]. The reference electrode $\mathrm{FPz}$ (CMS/DRL) is located on the forehead, the input electrodes are two front (left and right of the reference: AF7, AF8, silver made) and two posteriors, above each ear (TP9 and TP10, conductive silicone -rubber) (Figure 1). The device acquires signals at $256 \mathrm{~Hz}$ sampling frequency.

Raw EEG data were collected using the MUSE application [19], paired with a smartphone through Bluetooth Low Energy (BLE). However, the reliability of MUSE can be questioned. The limited number of electrodes can preclude the multi-networks

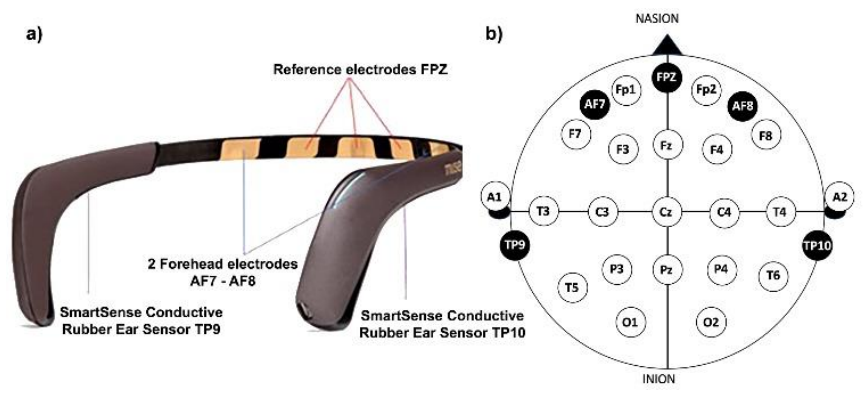

Figure 1. a) MUSE 2 headband sensors overview. b) Top-down view of the EEG electrode positions on the subject's head. 
evaluation from being focused on a specific area of the brain. The frontal electrodes are more prone to collect eye-blink and movement artifacts, and they can disrupt the measurements of actual brain waves [4]. Dry electrodes may also be more prone to result in discomfort over time and pose a higher risk of misplacement on the forehead, resulting in lower accuracy of the signal acquired. In addition, certain head shapes, head sizes, and hairstyles made data collection difficult, the poor contact with the head surface does not allow a proper data acquisition [20], [21]. Nevertheless, many studies demonstrated its ability to be applied in research experimental context. Krigolson et al. [18] demonstrated that MUSE can be used successfully for EventRelated Potential (ERP) study applications. They tested the reliability of ERP data collected with MUSE using a resampling analysis, obtaining reliable ERP components with MUSE (especially the N200) with a minimal number of participants. Youssef et al. [22], in their study for lies detection using MUSE, showed great success in their experimental purpose. Ratti and colleagues [23] compared EEG medical devices with consumer MUSE portable devices. This study demonstrated that MUSE PSDs were similar to medical systems, but with higher variation (the power spectral ratio was between 0.975 and 1.025 for medical equipment, ratios between 1.125 and 1.225 for MUSE). This broadband increase in the power spectrum of MUSE data may reflect artifacts in data recorded by dry electrodes. However, MUSE is simple to set up, the applicability is quick (less than 10 min) and simple, which is significantly convenient for the selfhelp applications.

\subsubsection{EEG data processing}

EEG recording is prone to collect noise and physiological artifacts, such as eye blinking, movements, and non-physiological artifacts such as electrical interference. Therefore, it is very essential to apply processing and denoising to the recorded EEG data. Based on the literature background, a processing custom code was implemented to filter and isolate the signal of interest: notch filter was used for removing the power line noise
$(50 / 60 \mathrm{~Hz})$, a high-pass filter at $0.1 \mathrm{~Hz}$ to remove DC offset and low-frequency skin potential artifacts, a low-pass filter at $45 \mathrm{~Hz}$ to remove high-frequency noise [24]. The independent component analysis (ICA), which decomposes the signal into maximally independent components and artifacts components, was applied. After a visual inspection, the eyeblink component was removed, and the data were reconstructed (Figure 2). Epochs of $2.000 \mathrm{~ms}$ with $1.000 \mathrm{~ms}$ overlap were extracted from artifacts-free continuous data. Then the power spectrum analysis (PSD) was computed using a pwelch function with Hamming 256-samples window, with $50 \%$ overlap. The output was normalized using the Z-score normalization method.

\subsubsection{EEG features extraction}

To establish the correlation between EEG frequency bands and the subjective thermal sensation, retrieved through a questionnaire, the features extraction was performed. The output of pre-processing step represents the five major brain waves in the different frequency ranges: delta, theta, alpha, beta, and gamma waves.

Once the five brain waves were computed the main EEG features were calculated, based on their relevance in the context of the thermal comfort assessment using EEGs.

\subsection{Experimental campaign}

\subsubsection{Participants}

Twenty-three healthy volunteers were enrolled for the experiment. They were informed about the experimental protocol and data management. Collected data were anonymized. Two experimental sessions were conducted during wintertime from January to February 2021, and summertime in July 2021. The selected group included 9 males and 14 females. All volunteers were local students at the "University of Perugia", where the experiment took place. None of them had a pathological history. Personal information was collected with a survey filled by all subjects at the end of each test. Table 1 summarizes the information about the participants involved in
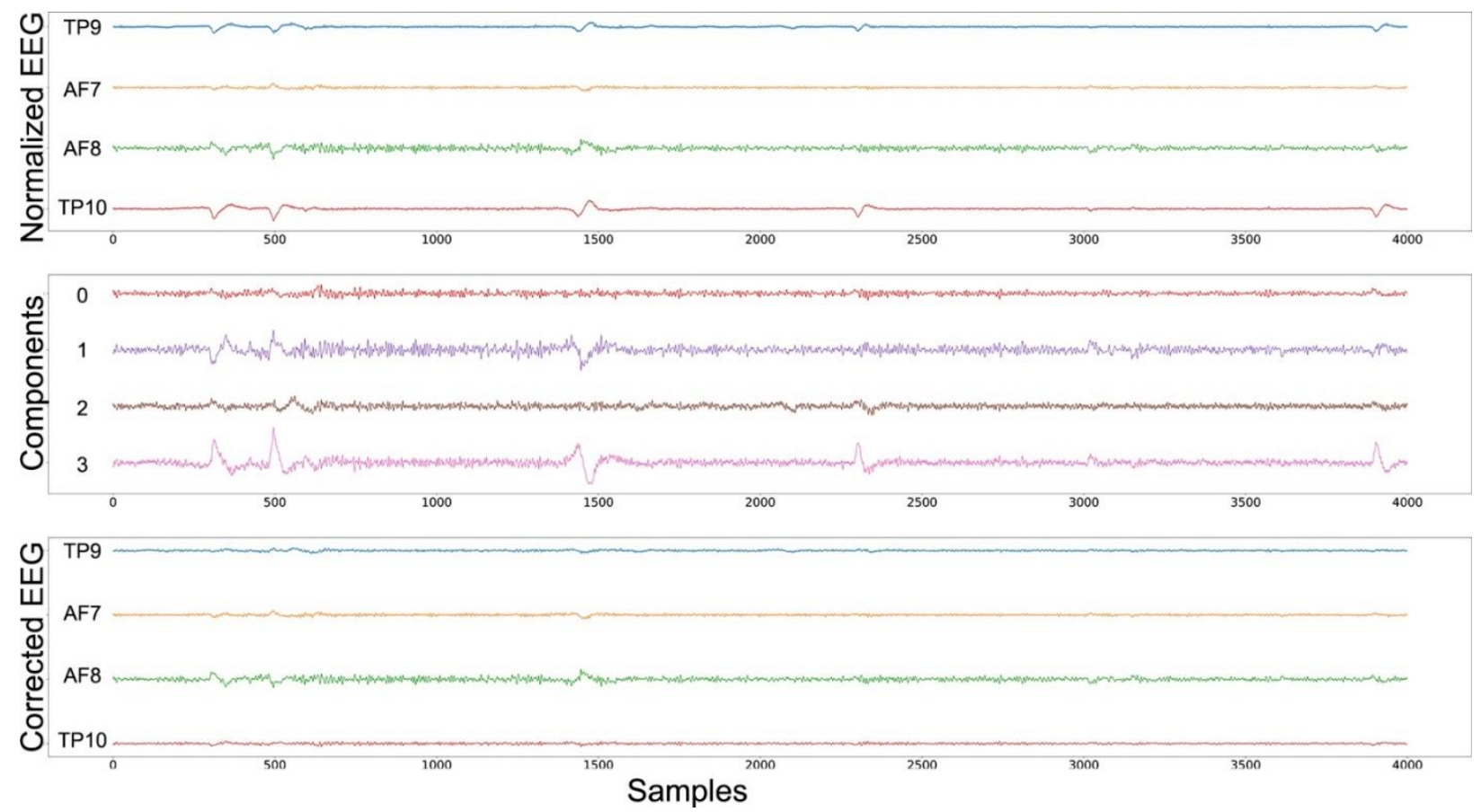

Figure 2. Normalized EEG data before eyeblink component removal; Corrected EEG data after eyeblink component removal and all the components estimated by ICA are reported. Component 3 was rejected. 
the campaigns: range of ages, mean and standard deviation (std) of height and weight. The average clothing thermal insulation was 1.1 clo and 0.43, typical for wintertime and summertime respectively. The metabolic rate of participants was 1.1 met according to standard ISO 7730 [25]. The second part of the survey aims at collecting data about thermal perception. In particular, the sensation vote for each thermal condition is given through a 5 -points scale going from -2 to 2 , where 0 corresponds to neutrality.

\subsubsection{Experimental setup: the controlled test room}

The experiments were carried out in the NEXT.ROOM $\left(4 \times 4 \times 2.7 \mathrm{~m}^{3}\right)$, a novel test room built up at the Engineering campus of Perugia University (Italy) for human-comfort studies. The NEXT.ROOM ambient temperature was controlled through the installed Heating, Ventilation \& Air Conditioning (HVAC) system based on a heat pump with an inverter and providing four levels of fan speed. The room has a window that was shaded during all the tests of the experimental campaign here described, while the internal level of illuminance was kept constant thanks to the installed artificial lighting system.

The environmental parameters inside the NEXT.ROOM were continuously monitored employing a fixed microclimate station located in the centre of the room and the associated data logging system [26]. Table 2 provides information about the sensors installed in the test room, their accuracies, and the environmental boundaries monitored during cold $\left(16{ }^{\circ} \mathrm{C}\right)$, neutral $\left(24^{\circ} \mathrm{C}\right)$, and warm $\left(31^{\circ} \mathrm{C}\right)$ tests accounting for both the winter and summertime series.

\subsubsection{Experimental setup: environmental condition}

During the experiments (both winter and summer seasons) the air velocity was always below $0.1 \mathrm{~m} / \mathrm{s}$, the relative humidity of air was not dependently controlled, while the air temperature was set at $16^{\circ} \mathrm{C}$ (cold), $24^{\circ} \mathrm{C}$ (neutral), and $31^{\circ} \mathrm{C}$ (warm) according to the thermal sensation perceived by the participants collected with the survey (Table 3).

A specific schedule for the experiment was adopted. The measurements were done from 10:00 a.m. to 1:00 p.m. and from 3:00 p.m. to 6:30 p.m. Subjects were asked to sit down and keep relaxed; no activity was allowed. The experimental procedure was carried out sequentially for 2 days for each thermal condition, in both seasons. Each experiment lasted $20 \mathrm{~min}, 15 \mathrm{~min}$ for thermal

Table 1. Details of the subjects participating in experiments.

\begin{tabular}{lc}
\hline Information & Details \\
\hline Total number of subjects & $23(9$ males and 14 females $)$ \\
Age (min-max) & $(27-32)$ years \\
Height (mean \pm std) & $(162 \pm 12) \mathrm{cm}$ \\
Mean of Weight $($ mean \pm std) & $(50.7 \pm 15.3) \mathrm{kg}$ \\
\hline
\end{tabular}

Table 2. Technical information of the sensors for environmental parameters monitoring

\begin{tabular}{lcc}
\hline Sensor & Environmental parameter & Accuracy \\
\hline Thermal-hygrometer & Air temperature, ${ }^{\circ} \mathrm{C}$ & $\pm 0.1{ }^{\circ} \mathrm{C}$ \\
& Relative Humidity, $\%$ & $\pm 1.5 \%$ \\
Black globe radiant & Mean radiant & $\pm 0.15{ }^{\circ} \mathrm{C}$ \\
temperature sensor & Temperature, ${ }^{\circ} \mathrm{C}$ & $\pm 0.05 \mathrm{~m} / \mathrm{s}$ \\
Hotwire anemometer & Air velocity, $\mathrm{m} / \mathrm{s}$ & $\pm 50 \mathrm{ppm}(+2 \%)$ \\
$\mathrm{CO}_{2}$ sensor & $\mathrm{CO}_{2}$ concentration, ppm & $\pm 5 \%$ \\
Luxmeter & Illuminance, lx & \\
\hline
\end{tabular}

Table 3. Mean values and std of environmental parameters monitored in the climate chamber during the experimental sessions.

\begin{tabular}{lccc}
\hline Measured Parameters & Cold & Neutral & Warm \\
\hline Air temperature, ${ }^{\circ} \mathrm{C}$ & $16.7 \pm 0.3$ & $24.3 \pm 0.5$ & $31.4 \pm 0.5$ \\
Relative humidity, \% & $25.9 \pm 0.5$ & $20.1 \pm 0.2$ & $18.2 \pm 0.4$ \\
Air velocity, $\mathrm{m} / \mathrm{s}$ & $0.1 \pm 0.02$ & $0.07 \pm 0.04$ & $0.09 \pm 0.03$ \\
Mean radiant temperature, ${ }^{\circ} \mathrm{C}$ & $16.4 \pm 0.6$ & $23.9 \pm 0.5$ & $30.2 \pm 0.4$ \\
$\mathrm{CO}_{2}$ concentration, ppm & $487 \pm 12$ & $492 \pm 4$ & $502 \pm 6$ \\
Illuminance, Ix & $287 \pm 22$ & $290 \pm 25$ & $281 \pm 23$ \\
\hline
\end{tabular}

adaptation (the previous study affirmed that people need $15 \mathrm{~min}$ to adapt to a new environment [27]), and 5 for recording data. (Figure 3).

\section{RESULTS}

The capability of discriminating the cold, warm and neutral sensations starting from the portable EEG measurements was evaluated in terms of statistical significance between brain waves features.

EEG features were divided into three groups (cold, neutral, and warm) according to the thermal sensation scores expressed by the participants in the questionnaire. Mean $(\bar{f})$ and standard uncertainty of the mean $\left(u_{f}\right)$ of each feature was then calculated according to [28].

The normality of each group of features was evaluated with the Shapiro test [29]. Given that all groups presented a nongaussian distribution, the statistical significance was determined with the non-parametric Kruskal-Wallies test [30]. Table 4 reports the features that turned out to provide a significant statistical difference ( $\mathrm{p}$-value $<0.05)$. The results showed how brain activities were altered by the thermal sensation perceived by occupants to the different environmental temperatures they were exposed to. In particular, the outcomes revealed that EEG features connected to high-frequency bands, such as beta and gamma for both frontal and temporal electrodes, tended to decrease with a warm sensation. Instead, features that express the mean power of low-frequency bands registered an opposite trend (Alpha-beta ratio AF8, Relative alpha TP9, Theta beta ratio AF8).

The capability of a feature to discriminate between two different thermal conditions was further investigated with a posthoc analysis based on the Dwass-Steel-Critchlow-Fligner pairwise comparison test [31]. Results from the test are reported in Table 5. The pairwise comparison results demonstrated that

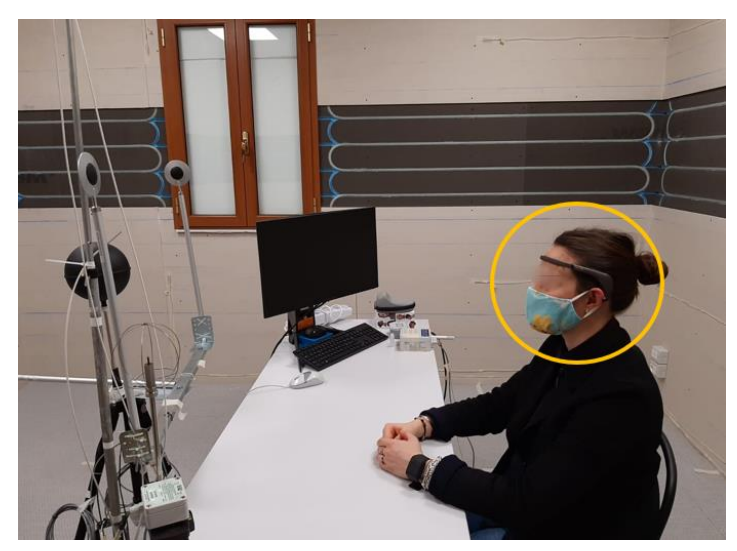

Figure 3. Measurements of EEG signals with Muse headband Interaxon in climate chamber. A facial mask was not used during the experiments. 
Table 4. Mean feature $(\bar{f})$, standard uncertainty of the mean $(u), \mathrm{H}$-statistic, and the significative p-value (p-value<0.05) for each of the statistically significant EEG features are reported.

\begin{tabular}{|c|c|c|c|c|c|}
\hline EEG features & $\begin{array}{c}\bar{f} \pm u_{f} \\
\text { (Cold) }\end{array}$ & $\begin{array}{l}\bar{f} \pm u_{f} \\
\text { (Neutral) }\end{array}$ & $\begin{array}{l}\bar{f} \pm u_{f} \\
\text { (Warm) }\end{array}$ & H-statistic & p-value \\
\hline Alpa beta ratio TP10 & $1.2 \pm 0.08$ & $1.3 \pm 0.07$ & $1.3 \pm 0.07$ & 3.3 & 0.04 \\
\hline Alpha beta product TP9 in dB & $1.3 \pm 0.12$ & $1.5 \pm 0.14$ & $1.6 \pm 0.10$ & 8.1 & 0.02 \\
\hline Alpha beta product AF8 in dB & $0.1 \pm 0.05$ & $0.1 \pm 0.03$ & $0.1 \pm 0.02$ & 8.2 & 0.02 \\
\hline Beta AF8 in dB & $0.4 \pm 0.10$ & $0.3 \pm 0.07$ & $0.1 \pm 0.06$ & 13.9 & 0.001 \\
\hline Beta TP9 in dB & $0.5 \pm 0.05$ & $0.0 \pm 0.09$ & $0.4 \pm 0.5$ & 5.3 & 0.006 \\
\hline Gamma AF7 in dB & $0.1 \pm 0.09$ & $0.1 \pm 0.05$ & $-0.1 \pm 0.09$ & 6.7 & 0.03 \\
\hline Gamma AF8 in dB & $0.1 \pm 0.1$ & $0.0 \pm 0.06$ & $-0.1 \pm 0.07$ & 12.4 & 0.002 \\
\hline Gamma TP10 in dB & $0.2 \pm 0.06$ & $0.2 \pm 0.02$ & $0.1 \pm 0.04$ & 7.9 & 0.02 \\
\hline Gamma TP9 in dB & $0.2 \pm 0.07$ & $0.1 \pm 0.05$ & $0.1 \pm 0.05$ & 8.9 & 0.01 \\
\hline Relative Alpha TP9 & $0.3 \pm 0.17$ & $0.3 \pm 0.02$ & $0.3 \pm 0.07$ & 10.3 & 0.006 \\
\hline Relative Beta AF8 & $0.3 \pm 0.06$ & $0.3 \pm 0.05$ & $0.1 \pm 0.07$ & 16.9 & $<0.001$ \\
\hline Relative Gamma TP10 & $0.1 \pm 0.02$ & $0.1 \pm 0.01$ & $0.1 \pm 0.02$ & 6.1 & 0.04 \\
\hline Relative Gamma TP9 & $0.1 \pm 0.03$ & $0.0 \pm 0.02$ & $0.0 \pm 0.03$ & 4.8 & 0.09 \\
\hline Relative Theta AF8 & $0.1 \pm 0.06$ & $0.1 \pm 0.05$ & $0.2 \pm 0.07$ & 9.2 & 0.01 \\
\hline $\begin{array}{l}\text { Temporal Asymmetry Alpha in } \\
d B\end{array}$ & $0.1 \pm 0.03$ & $0.0 \pm 0.02$ & $0.0 \pm 0.02$ & 4.5 & 0.01 \\
\hline $\begin{array}{l}\text { Temporal Asymmetry Delta in } \\
\mathrm{dB}\end{array}$ & $0.1 \pm 0.03$ & $0.0 \pm 0.03$ & $0.0 \pm 0.02$ & 6.7 & 0.03 \\
\hline $\begin{array}{l}\text { Temporal Asymmetry Theta in } \\
\mathrm{dB}\end{array}$ & $0.1 \pm 0.03$ & $0.0 \pm 0.4$ & $0.0 \pm 0.03$ & 8.7 & 0.01 \\
\hline Theta beta ratio AF8 & $-0.3 \pm 0.71$ & $0.1 \pm 0.24$ & $2.3 \pm 2.31$ & 7.2 & 0.02 \\
\hline
\end{tabular}

all the features (except for Alpha-beta ratio TP9, Beta TP10, and Gamma TP9) were different between cold and warm thermal sensations. Beta AF8, Gamma AF8, and Relative beta AF8 also showed differences between neutral and warm conditions, as shown in Figure 4. None of the measured features showed differences between cold and neutral thermal sensations.

In general, EEG measurements performed with the portable device showed a correlation with the thermal sensations in terms of increase or decrease of power of brain waves. The warm

Table 5. Dwass-Steel-Critchlow-Fligner pairwise comparison between cold (c), neutral (n), and warm (w) thermal sensation results ( $*$ for $p$-value $<0.05)$.

\begin{tabular}{|c|c|c|c|}
\hline EEG features & $\begin{array}{c}c-n \\
\text { (p-value) }\end{array}$ & $\begin{array}{c}c-w \\
\text { (p-value) }\end{array}$ & $\begin{array}{c}n-w \\
\text { (p-value) }\end{array}$ \\
\hline Alpa beta ratio TP10 & 0.3 & 0.1 & 0.7 \\
\hline Alpha beta product TP9 in dB & 0.2 & $0.01 *$ & 0.6 \\
\hline Alpha beta product AF8 in $\mathrm{dB}$ & 0.5 & $0.02 *$ & 0.1 \\
\hline Beta AF8 in $\mathrm{dB}$ & 0.5 & $0.001^{*}$ & $0.03^{*}$ \\
\hline Beta TP9 in dB & 0.1 & $0.02 *$ & 0.9 \\
\hline Gamma AF7 in dB & 0.2 & $0.04 *$ & 0.6 \\
\hline Gamma AF8 in dB & 0.5 & $0.003^{*}$ & $0.04 *$ \\
\hline Gamma TP10 in dB & 0.5 & $0.02 *$ & 0.3 \\
\hline Gamma TP9 in dB & 0.1 & 0.2 & 0.8 \\
\hline Relative Alpha TP9 & 0.1 & $0.004^{*}$ & 0.5 \\
\hline Relative Beta AF8 & 0.9 & $0.004^{*}$ & $0.001 *$ \\
\hline Relative Gamma TP10 & 0.4 & $0.04 *$ & 0.6 \\
\hline Relative Gamma TP9 & 0.2 & $0.02 *$ & 0.3 \\
\hline Relative Theta AF8 & 0.6 & 0.2 & $0.007^{*}$ \\
\hline Temporal Asymmetry Alpha in dB & 0.5 & $0.02 *$ & 0.2 \\
\hline Temporal Asymmetry Delta in dB & 0.3 & $0.02 *$ & 0.6 \\
\hline Temporal Asymmetry Theta in dB & 0.6 & $0.01^{*}$ & 0.2 \\
\hline Theta beta ratio AF8 & 0.2 & $0.03^{*}$ & 0.4 \\
\hline
\end{tabular}

sensation could be correlated to an increase of alpha and theta waves, indicating that when subjects are exposed to a warm environment tend to be less concentrated and unable to keep focused. On the other hand, in cold conditions, there is an increase in the activity of beta and gamma waves, which are the main brain waves connected to high mental activity. At the same time, high levels of beta and gamma can be synonyms of high stress, indicating that the cold condition is perceived as more stressful than the warm one. Results obtained with the MUSE turned out to be aligned with the state of the art concerning thermal comfort measurements based on EEG data.

\section{CONCLUSIONS}

As it is already known the prominence of an EEG frequency band is correlated to a certain type of mental state. For example, the high power of gamma waves corresponds to high mental activity, vice versa dominant alpha corresponds to relaxed conditions. Some studies revealed that EEG theta waves increased while beta waves decreased with a comfortable thermal state, others showed that high values of theta band correspond to a high state of arousal level and vice versa. All studies have been performed with medical and non-portable devices, providing accurate EEG measurements, but with poor wearability and not applicable in real-life situations. For this reason, the proposed study aims at demonstrating whether a lowcost portable EEG device (MUSE) could be used to perform thermal comfort measurements, providing better wearability but with lower measurement accuracy. The experiments were performed in a controlled environment, where EEG was measured on 23 subjects exposed to warm, neutral, and cold conditions. Considering the different thermal sensations perceived by subjects exposed to the same environmental condition, before performing the statistical analysis, the physiological features were divided and classified according to the thermal sensation questionnaire-based results into cold, 
Figure 4. The main representative Kruskal-Wallies results. Mean $(\overline{\boldsymbol{f}})$ and standard uncertainty of the mean $\left(\boldsymbol{u}_{\boldsymbol{f}}\right)$ in each condition are shown.
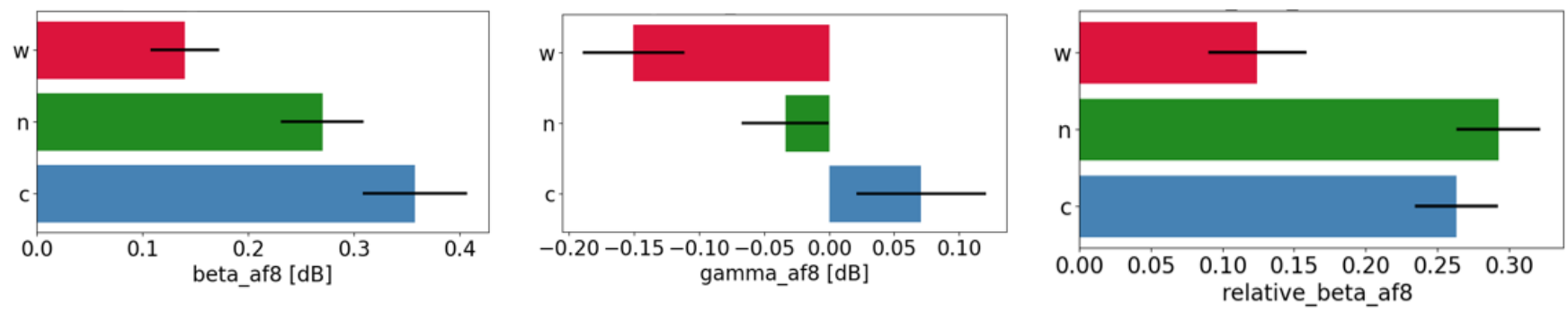

neutral, and warm groups. This approach allows investigating the thermal perception of occupants overcoming the limits due to the different nationalities and personal thermal history, focusing on just the perception felt by the subjects during experiments. Results showed that all the EEG features calculated presented a statistical difference between cold and warm sensations. Beta AF8, Gamma AF8 and, Relative beta AF8 showed significant differences also between neutral and warm sensations. In general, the results demonstrate that MUSE EEG wearable device could be used for thermal comfort measurements, discriminating cold and warm thermal sensation.

Due to its comfortable design, the MUSE considerably reduces the measurement's intrusiveness, reducing consequently the time of application and discomfort due to wearability. The presence of dry electrodes facilitates the recording procedure but, the absence of a conductivity gel increases the electrical impedance between the dry electrodes and the skin, making the devices more prone to register artifacts, such as muscular movements and eyeblink, with the risk of reducing the signal-to-noise ratio. Therefore, signal processing for data cleaning and artifacts removal, based on the approach proposed in this paper, is required. In addition, the usage of water solution, to improve the conductivity of MUSE electrodes during its application, could introduce a further source of uncertainty in the case of longlasting experiments. The water evaporation can lead to drifts of the measured voltage, resulting in a misleading features interpretation. For longer experiments, future investigations are required to estimate the measurement uncertainty in the case of the usage of portable EEG sensors for real-life applications.

Nevertheless, for future developments, the idea to study the EEG signal in conjunction with other physiological signals, such as ECG, ST, and GSR has been considered. This approach could allow the generation of comprehensive knowledge about the physiological response of the mechanisms involved in the human thermoregulatory system. New experimental campaigns will be conducted, increasing the number of samples to give consistency to the statistical results. An investigation about how the gender and age of subjects can affect thermal perception will be considered, as an additional aspect of the study. The final scope is to identify the most relevant physiological features to be used as input for the creation of predictive models, based on ML techniques, capable of thermal comfort level classification.

\section{ACKNOWLEDGMENT}

The research has been co-founded by the Italian Ministry of Research through the NEXT:COM (Prot.20172FSCH4) "Towards the NEXT generation of Multiphysics and multidomain environmental COMfort models: theory elaboration and validation experiment" project, within the PRIN 2017 program.

\section{REFERENCES}

[1] Q. Zhao, Z. Lian, D. Lai, Thermal comfort models and their developments: A review, Energy Built Environ., vol. 2, no. 1, pp. 21-33, Jan. 2021.

DOI: $10.1016 /$ J.ENBENV.2020.05.007

[2] P. O. Fanger, Assessment of man's thermal comfort in practice, Br. J. Ind. Med., vol. 30, no. 4, 1973, pp. 313-324.

DOI: $\underline{10.1136 / O E M .30 .4 .313}$

[3] J. F. Nicol, M. A. Humphreys, Adaptive thermal comfort and sustainable thermal standards for buildings, Energy Build., vol. 34, no. 6, pp. 563-572, 2002.

DOI: $\underline{10.1016 / \mathrm{S} 0378-7788(02) 00006-3}$

[4] Silvia Angela Mansi, Giovanni Barone, Cesare Forzano, Ilaria Pigliautile, Maria Ferrara, Anna Laura Pisello, Marco Arnesano, Measuring human physiological indices for thermal comfort assessment through wearable devices: A review, Meas. J. Int. Meas. Confed., vol. 183, p. 109872, 2021. DOI: $10.1016 /$ i.measurement.2021.109872

[5] Y. Yao, Z. Lian, W. Liu, Q. Shen, Experimental study on physiological responses and thermal comfort under various ambient temperatures, Physiol. Behav., 2008. DOI: $10.1016 /$ i.physbeh.2007.09.012

[6] J. Gwak, M. Shino, K. Ueda, M. Kamata, An investigation of the effects of changes in the indoor ambient temperature on arousal level, thermal comfort, and physiological indices, Appl. Sci., 2019. DOI: $10.3390 /$ app 9050899

[7] S. Liu, S. Schiavon, H. Prasanna Das, M. Jin, C. J. Spanos, Personal thermal comfort models with wearable sensors, Build. Environ., Vol 162, 2019.

DOI: $10.1016 /$ j.buildenv.2019.106281

[8] P. Arpaia, N. Moccaldi, R. Prevete, I. Sannino, A. Tedesco, A Wearable EEG Instrument for Real-Time Frontal Asymmetry Monitoring in Worker Stress Analysis, IEEE Trans. Instrum. Meas., vol. 69, no. 10, pp. 8335-8343, 2020. DOI: $10.1109 /$ TIM.2020.2988744

[9] J. Satheesh Kumar, P. Bhuvaneswari, Analysis of Electroencephalography (EEG) Signals and Its Categorization-A Study, Procedia Engineering, Volume 38, 2012.

DOI: $10.1016 /$ i.proeng. 2012.06 .298

[10] U. Herwig, P. Satrapi, C. Schönfeldt-Lecuona, Using the International 10-20 EEG System for Positioning of Transcranial Magnetic Stimulation, Brain Topogr., vol. 16, no. 2, pp. 95-99, Dec. 2003.

DOI: 10.1023/B:BRAT.0000006333.93597.9D

[11] Lv B, Su C, Yang L, Wu T. Effects of stimulus mode and ambient temperature on cerebral responses to local thermal stimulation: An EEG study. Int J Psychophysiol. 2017 Mar; 113:17-22 DOI: 10.1016/j.ijpsycho.2017.01.003

[12] Lim, J. R., G. H. Baek and E. Jeon. Analysis of the Correlation between Thermal Sensations and Brain Waves via EEG Measurements. 2018.

[13] Son, Young Joo \& Chun, Chungyoon. Research on Electroencephalogram (EEG) to measure Thermal Pleasure in Thermal Alliesthesia in Temperature Step-change Environment. 2018. Indoor Air. 28. 10.1111/ina.12491 DOI: $\underline{10.1111 / \text { ina. } 12491}$ 
[14] Wu M, Li H, Qi H. Using electroencephalogram to continuously discriminate feelings of personal thermal comfort between uncomfortably hot and comfortable environments. Indoor Air. 2020 May;30(3):534-543

DOI: $10.1111 /$ ina.12644

[15] Zhu, M., Liu, W. \& Wargocki, P. Changes in EEG signals during the cognitive activity at varying air temperature and relative humidity. J Expo Sci Environ Epidemiol 30, 285-298 (2020) DOI: $\underline{10.1038 / s 41370-019-0154-1}$

[16] Sci-Hub, Metrological characterization of a low-cost electroencephalograph for wearable neural interfaces in industry 4.0 applications. 2021 IEEE International Workshop on Metrology for Industry 4.0 \& IoT (MetroInd4.0\&IoT), Rome, Italy, 7-9 June 2021

DOI: $10.1109 /$ metroind4.0iot51437.2021.9488445

[17] J. LaRocco, M. D. Le, D. G. Paeng, A Systemic Review of Available Low-Cost EEG Headsets Used for Drowsiness Detection, Front. Neuroinform., vol. 14, Oct. 2020, p. 42 DOI: 10.3389/FNINF.2020.553352/BIBTEX

[18] O. E. Krigolson, C. C. Williams, A. Norton, C. D. Hassall, F. L. Colino, Choosing MUSE: Validation of a low-cost, portable EEG system for ERP research, Front. Neurosci., 2017.

DOI: $10.3389 /$ fnins.2017.00109

[19] Mind monitor. Online [Accessed 16 December 2021] https://mind-monitor.com/

[20] E. S. Kappenman, S. J. Luck, The effects of electrode impedance on data quality and statistical significance in ERP recordings, Psychophysiology, vol. 47, no. 5, 2010, pp. 888-904. DOI: $10.1111 / \mathrm{i} .1469-8986.2010 .01009 . \mathrm{x}$

[21] X. Wang, D. Li, C. C. Menassa, V. R. Kamat, Investigating the effect of indoor thermal environment on occupants' mental workload and task performance using electroencephalogram, Build. Environ., vol. 158, no. March, 2019, pp. 120-132. DOI: $10.1016 /$ i.buildenv.2019.05.012

[22] A. E. Youssef, H. T. Ouda, M. Azab, MUSE: A Portable Costefficient Lie Detector, in 2018 IEEE 9th Annual Information Technology, Electronics and Mobile Communication Conference, IEMCON 2018, 2019.

DOI: $\underline{10.1109 / \text { IEMCON.2018.8614795 }}$
[23] E. Ratti, S. Waninger, C. Berka, G. Ruffini, A. Verma, Comparison of medical and consumer wireless EEG systems for use in clinical trials, Front. Hum. Neurosci., 2017. DOI: $10.3389 /$ fnhum. 2017.00398

[24] W. Peng, EEG preprocessing and denoising, EEG Signal Process. Featur. Extr., Jan. 2019, pp. 71-87. DOI: $10.1007 / 978-981-13-9113-25$

[25] ISO 7730:2005-Ergonomics of the Thermal Environment Analytical Determination and Interpretation of Thermal Comfort Using Calculation of the PMV and PPD Indices and Local Thermal Comfort Criteria.

[26] I. Pigliautile, S. Casaccia, N. Morresi, M. Arnesano, A. L. Pisello, G. M. Revel, Assessing occupants' personal attributes in relation to human perception of environmental comfort: Measurement procedure and data analysis, Build. Environ., 2020. DOI: $10.1016 /$ i.buildenv.2020.106901

[27] A. Ghahramani, G. Castro, B. Becerik-Gerber, X. Yu, Infrared thermography of human face for monitoring thermoregulation performance and estimating personal thermal comfort, Build. Environ., vol. 109, 2016, pp. 1-11. DOI: $10.1016 /$ i.buildenv.2016.09.005

[28] ISO, Evaluation of measurement data - Guide to the expression of uncertainty in measurement, Int. Organ. Stand. Geneva ISBN, vol. 50, no. September, p. 134, 2008. Online [Accessed 16 December 2021] https://www.bipm.org/en/publications/guides

[29] M. Brzezinski, The Chen-Shapiro test for normality, Stata J., vol. 12, no. 3, Sep. 2012, pp. 368-374. DOI: $10.1177 / 1536867 X 1201200302$

[30] E. Ostertagová, O. Ostertag, J. Kováč, Methodology and application of the Kruskal-Wallis test, Appl. Mech. Mater., vol. 611, 2014, pp. 115-120. DOI: 10.4028/WWW.SCIENTIFIC.NET/AMM.611.115

[31] D. E. Critchlow, M. A. Fligner, On distribution-free multiple comparisons in the one-way analysis of variance, vol. 20, no. 1, Jan. 2007, pp. 127-139.

DOI: $\underline{10.1080 / 03610929108830487}$ 